\title{
Sistemas de informação em contexto de mudança organizacional: uma reflexão sobre suas potencialidades
}

\author{
Odibar João Lampeão - ojpeao@gmail.com ${ }^{1}$ \\ Marilia Barreto de Santana - mariliabsantana@gmail.com²
}

\begin{abstract}
Resumo - Os dias de hoje são marcados por avanços tecnológicos e volumes de informações crescentes, que afetam às organizações e suas operações. Para responder eficazmente face a diversas demandas, as organizações buscam sistemas de informação, incontornavelmente baseados em artefactos computacionais, para que não fiquem atrás na esfera da competição e subsistência. No entanto, também nesse contexto, rotinas organizacionais, padrões de actividades e processos, precisam serem planificados ou revistos em adequações para que ocorram mudanças desejadas. Com este ensaio, baseado em pesquisa documental, pretende-se relacionar aspectos que ajudem a compreender e considerar que os sistemas de informação se assumem como elemento fundamental para as organizações, especialmente, num contexto de perenes mudanças.
\end{abstract}

Palavras-chave: Sistemas de informação; Mudança organizacional; Rotinas.

\section{Information systems in the context of organizational change: a look at their potentialities}

\begin{abstract}
Nowadays, the days are marked by technological advances and increasing volumes of information which affect organizations and their operations. To respond effectively to diverse demands, organizations seek information systems, inevitably based on computational artifacts, so that they do not fall behind in the sphere of competition and subsistence. However, also in this context, organizational routines, patterns of activities and processes, need to be planned or revised in order to make the desired changes. This essay, based on documentary research, aims to relate aspects that help to understand and consider that information systems are assumed as a fundamental element for organizations, especially in a context of perennial changes.
\end{abstract}

Keywords: Information systems; Organizational change; Routines. 


\section{INTRODUÇÃO}

Durante muito tempo, as rotinas marcaram o quotidiano das organizações, não sendo pertinente a busca de análises mais aprofundadas para que se pudesse reagir melhor ao sentimento de mudança ou a própria mudança no seio das organizações. As rotinas aparecem, então, vistas como mecanismo de análise e controle (BECKER, 2004; MIETTINEN; VIRKKUNEN, 2005; MILAGRES, 20II), de redução de incertezas e manutenção da inercia (GRANT, I999; MÜLLER; VIEIRA, I999; BEUREN; MACOHON, 20II) e recentemente, como elemento que proporciona a própria a flexibilidade e a mudança (PENTLAND; FELDMAN, 2005; MIETTINEN; VIRKKUNEN, 2005; NASCIMENTO; RUAS; SILVA, 20I7).

Quase que paradoxalmente, como se depreende de alguns autores (SPULDARO, 20I3; STANCZYK-HUGIET, 20I4; HOWARD-GRENVILLE E RERUP, 20I6), as rotinas estão intrinsecamente ligadas a processos organizacionais que, com o andar do tempo, perante as realidades vivenciadas, necessitam de ser melhorados ou adequados, para permitir melhor resposta da organização ao ambiente. E essa abordagem encontrada em Suddaby e Foster (20I7), ao salientarem que com o tempo a organização se ossifica, apresentando, como os humanos, experiência, tradições e práticas adquiridas, que criam forças poderosas de inércia interna, precisando, então, de melhorar suas rotinas e processos possivelmente envelhecidos, de modo a materializar uma mudança que assegure sua sobrevivência.

Nas ultimas décadas as mudanças no trabalho e na estrutura organizacional foram vertiginosas, levando a que estruturas rígidas e hierarquizadas de outrora fossem substituídas pelos trabalhos em equipes, ao mesmo tempo que processos organizacionais tornaram-se mais dependentes da tecnologia que, por sua vez, evoluiu numa velocidade ímpar, solicitando apropriado acompanhamento (DELLAGNELO; MACHADO-DA-SILVA, 2000; BRESSAN, 2004)

Assiste-se um momento reputado como era do conhecimento e da informação (FEY, 20I2; SISSON; RYAN, 20I7) ou da informação digital (RAMALHO, 20I3; HENFRIDSSON, MATHIASSEN; SVAHN, 20I4) em que para as organizações, trabalhar com informação não é uma questão de escolha, mas sim de alinhamento com o contexto em que, o contrário, implica, com maior probabilidade, o degelo da organização.

Assim, estando numa era com ambientes internos e externos dinamicamente interconectados, é solicitada a organização maior habilidade para a mudança, apresentando-se sempre pronta para um monitoramento mais constante do contexto, embasando-se em informações sempre oportunas, onde possa identificar problemas e solucioná-los atempadamente (FONSECA; DOMINGUES, 20I7), como se propõe na figura I. 
Figura 1. Contexto de mudanças organizacionais

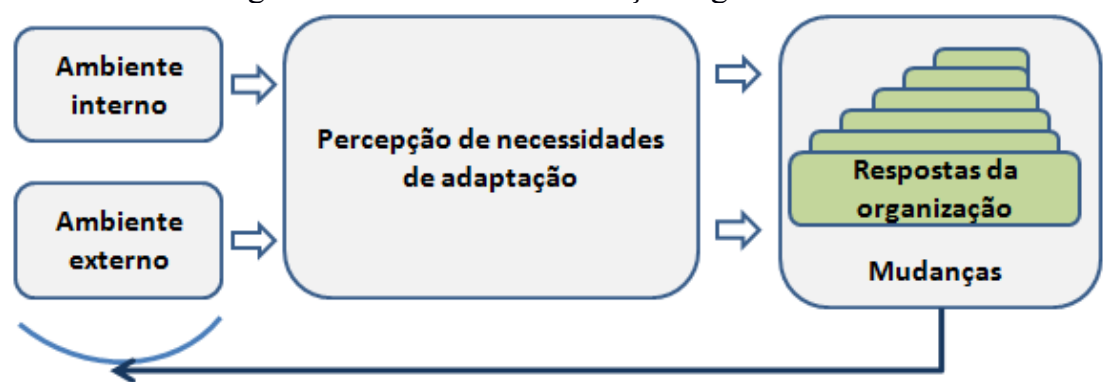

Fonte: Baseado em Bressan (2004); Carnall (2007) e Fonseca e Domingues (20I7).

O momento solicita que a tecnologia da informação, como ferramenta gerencial, deva ser utilizada para análise de dados, transformando-os em informações realmente úteis aos negócios das organizações (HENDERSON; VENKATRAMAN, I999; VOLKOW, 20I7). Assim, à medida que as empresas converterem dados em informações, modificarão, necessariamente, seus processos de decisão, a sua estrutura administrativa e a sua maneira de trabalhar, na qual decisões oportunistas transformar-se-ão em diretrizes e pressupostos estratégicos (MARKUS; ROBEY, I988; BORGES, I995; NETO; DORNELAS, 20I4).

Falar de mudança nas organizações é, basicamente, de entre outras coisas, falar de capacidade de resposta as demandas do meio (interno e externo) com objetivo de aumento ou manutenção do desempenho organizacional com base em uma estratégia (BRESSAN, 2004; CARNALL, 2007). E conforme Anderson (2017) apresenta, adaptar estratégias de mudança que obtenham sucesso envolve compreender aspetos do ambiente, dos indivíduos e da organização como um todo, ao mesmo tempo que se busca adequar a tecnologias para dinamizar processos organizacionais.

Tendo em conta a ideia de mudança organizacional e as afirmações recorrentes de que se presencia uma era da informação, onde o recurso a artefactos computacionais tem sido regra para dinamizar processos organizacionais, o presente ensaio procura buscar, através da visitação da literatura, elementos que exaltem o papel dos sistemas de informação nesses processos de mudanças organizacionais, passando por caracterizar primeiro os sistemas de informação, seguido da mudança organizacional, para findar com o relacionamento destas duas valências e levantar considerações inerentes.

\section{OS SISTEMAS DE INFORMAÇÃO}

O fato de se estar atualmente em uma sociedade da informação, remete a existência de diversos dados e informações que as organizações terão que coletar e perceber para melhor valor obter delas, gerando o conhecimento (REZENDE, 2002; OLIVEIRA; OLIVEIRA; LIMA, 2016), que perfaz a consciência e a compreensão de um conjunto de informações e os modos como essas informações podem ser uteis para apoiar uma tarefa específica ou para chegar a uma decisão (ABBASI; SARKER; CHIANG, 2016; STAIR; REYNOLDS, 2017). Desta feita, o conhecimento que se gera, ronda em torno de, dentre outras valências, questões como "saber que", "saber o quê", "saber quem", "saber onde", "saber quando", "saber o porquê" e "saber como" (SISSON; RYAN, 2017). 
Para ter o conhecimento, organizações precisam ter um ou mais sistemas de informação que lhes proporcionam dados e informações, bem como a interação com suas bases de dados (LAUDON; LAUDON, 2014; SILVA, ARAUJO; DORNELAS, 2018), come se sugere na figura 2. Esses sistemas de informação são um conjunto de componentes interrelacionados trabalhando juntos para coletar, recuperar, processar, armazenar e distribuir informação com a finalidade de facilitar o planeamento, o controle, a coordenação, a análise e o processo decisório em empresas e outras organizações, não sendo, necessariamente, dependentes de artefactos computacionais (LAUDON; LAUDON, 2014; LÖBLER, et al., 2014).

Figura 2. Acumulação de dados passado pelos sistemas de informação

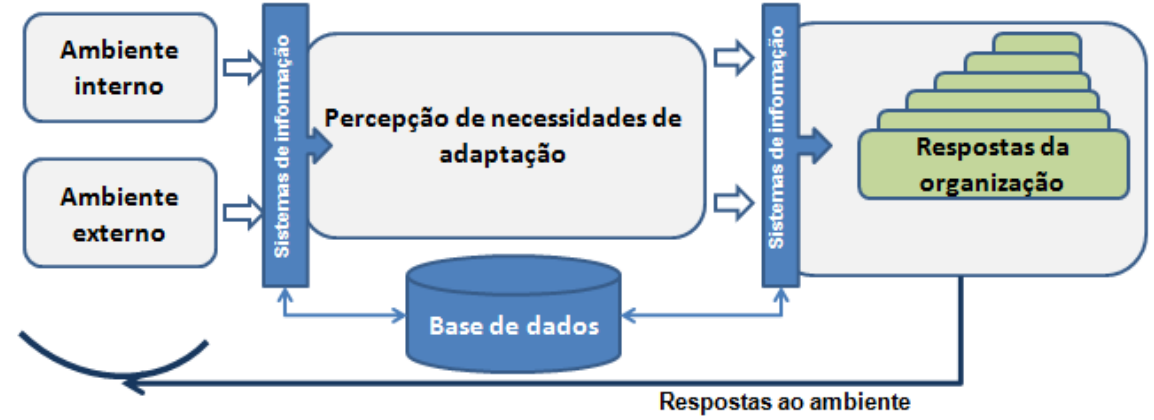

Fonte: Baseado em Abbasi, Sarker e Chiang (2016), Fonseca e Domingues (2017) e Stair e Reynolds (2017),

Como já refere Rezende (2006), os sistemas que manipulam e geram informação, usando ou não recursos tecnológicos, podem ser considerados como sistemas de informação. No entanto, o andar do tempo proporcionou a existência de massa de dados para armazenar e recuperar cada vez maiores, exigindo a transformação dos sistemas de informação manuais em sistemas processados eletronicamente, visando aos melhores resultados organizacionais em termos de custo-eficácia (FACCHINI; VARGAS, I992; LÖBLER, et al., 20I4).

Nessa vertente, Behl, O’brien e Marakas (2019) referem que os sistema de informação perfazem um conjunto organizado de pessoas, hardware, software, redes de comunicação e recursos de dados que coleta, transforma e dissemina informações em uma organização. E o seu conceito acaba por aclarar que, para estes, já não se afigura coerente, falar de sistemas de informação, sem referir sobre artefactos computacionais ou em tecnologia de informação.

Posto deste jeito, acentua-se a colocação de Olszak, Bartuś e Lorek (20I8) segundo a qual, o uso de tecnologias de informação tornam apta a organização para aceder e processar informação com maior rapidez e eficácia, impactando positivamente nos resultados da organização, ao mesmo tempo que propicia um aprendizado e aquisição de conhecimento.

De acordo com Stair e Reynolds (20I7), associando-se sistemas de informação e tecnologia de informação, chega-se à uma abordagem de processamento de informação que, para alcançar o objetivo, passa pelas seguintes fases: entrada (captação e reunião dos dados brutos para serem processados), processamento (transformação de dados em resultados úteis), saída (produção de informações úteis) e realimentação (utilização da informação originada do sistema para fazer mudanças na entrada ou nas atividades de processamento). 
Mas Belle, Eccles e Nash (20I0) já frizavam que sistema de informação não era simplesmente sobre os computadores. Para estes autores é sobre como as organizações podem fazer o melhor uso das tecnologias disponíveis e do entendimento de negócio para fornecer as informações necessárias para atingir seus objetivos.

Assim, ao se abordar questões de sistemas de informação, na maioria das vezes não existirá uma única resposta correta. A percepção do papel dos sistemas de informação poderá ajudar a que se escolha melhor o sistema e como ele poderá ajudar a organização em momentos de mudanças.

\subsection{O papel dos Sistemas de informação nas organizações}

A oferta de informações está cada vez mais recheada de dados de diversa natureza, solicitando que um ou mais mecanismos de controle, selecção e processamento sejam ativados para garantir que se faça uso do essencial e de valor acrescentado para a organização, tomando-se, consequentemente, melhores decisões.

Perante este cenário, pressionadas a atender novas demandas e a incorporar novas tecnologias, cada vez mais a organização da informação, desde a sua seleção, estruturação, recuperação, torna-se uma arma estratégica para auxiliar organizações na tomada de decisões e no alcance dos objetivos (FACCHINI; VARGAS, I992; LAUDON; LAUDON, 20I4), significando que, os sistemas de informação são uteis em toda a cadeia de suprimento das organizações e vêm provocando investimentos em infraestruturas de tecnologias de informação para o efeito (VIET; BEHDANI; BLOEMHOF, 20I8).

Diz-se então, que a necessidade de sistemas de informação nas empresas surgiu devido ao grande e crescente volume de informações que a organização possui, sendo que, a exigência do mercado competitivo, dinâmico e, principalmente, globalizado motivou as organizações a operarem com sistemas de informação eficientes, garantindo níveis mais elevados de produtividade e eficácia (BAZZOTTI; GARCIA, 2006; BEHL; O’BRIEN; MARAKAS, 20I9).

Conhecendo as potencialidades dos sistemas de informação, Boncompte (20I8) refere que muitas organizações os institucionalizam para permitir pagar-se por informação útil (que o autor chama de perfeita) e que possibilitem decisões estratégicas perante a concorrência. Mas certas organizações vão além e criam departamentos especializados para gestão e governança da informação e sistemas de informação que acabam sendo o canal para os tomadores de decisão se apetrecharem de dados e informações relevantes para respostas adequadas da organização (CAPEÇA; SÁ-SOARES, 20I5; PEARLSON; SAUNDERS; GALLETTA, 20I6), conforme se ilustra na figura 3 . 
Figura 3. Gestão de informação via departamentos especializados

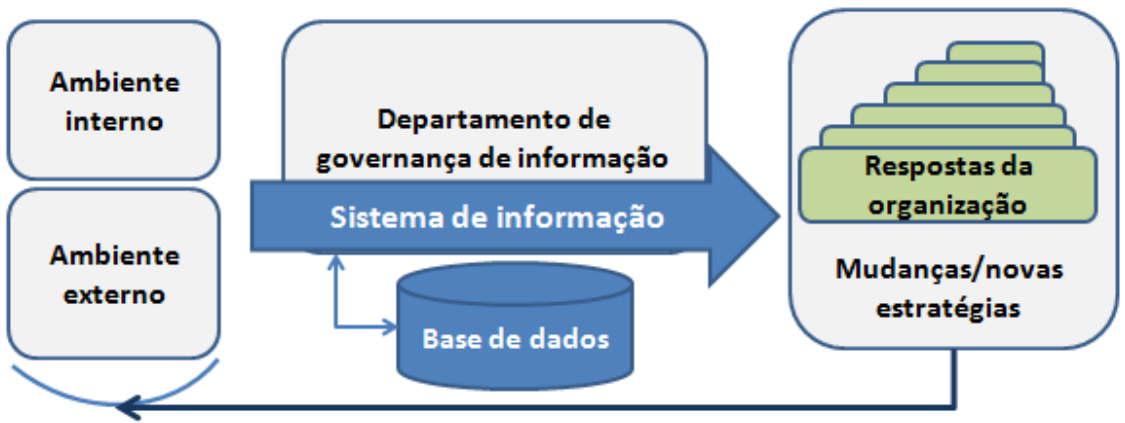

Fonte: Capeça, Sá-Soares (20I5), Pearlson, Saunders, Galletta (20I6) e Boncompte (20I8).

Assim, para que os sistemas de informação implementados sejam efetivos, estes devem corresponder as expetativas como: atender as reais necessidades dos usuários com presteza; apresentar custos compatíveis; adaptar-se constantemente às novas tecnologias da organização e; estar alinhados com as estratégias da organização (PEREIRA; FONSECA, I997; VASCONCELOS, et al., 2002; PEARLSON, SAUNDERS; GALLETTA, 20I6):

Permite-se então, em primeira instância, aceitar que o objetivo dos sistema de informação nas organizações é garantir o fluxo de dados e provir os meios de suporte essenciais para o fluxo de informação, sendo que o mesmo suporta, de forma integrada, a entrada e registo de dados da empresa, o processamento de dados, a produção de informação, a geração de relatórios e as necessidades de saída de dados (STAIR; REYNOLDS, 20I7).

Contudo, em segunda instancia, Rezende e Abreu (2013)indicam que independendo do nível ou classificação, o maior objetivo de um sistema de informação é o de apoiar a tomada de decisão, sendo que, caso não fite esse objetivo, sua existência pouco significado terá para a organização.

Posto desta forma, os sistemas de informação acabam por apresentar como papeis fundamentais, além da coleta de dados e informações no seu ambiente, o suporte de processos e operações organizacionais, suporta da tomada de decisões em tempo oportuno e suporte de estratégias em busca de vantagens competitivas (KASEMSAP, 20I8; BEHL; O'BRIEN; MARAKAS, 20I9).

É importante referir que para desempenhar seu papel, os sistemas de informação são constituídos por vários subsistemas, responsáveis por subgrupos de necessidades de informação e operação na organização, possuindo seus próprios fluxos de dados e suportando fluxos de informações específicas (STAIR; REYNOLDS, 20I7).

Nessa vertente, Askenäs e Westelius (2003) propõem cinco papéis principais para os sistemas de informação nas organizações: o manipulador (permitindo mudar ou conservar os processos de trabalho garantindo que não sejam destinados de forma diferente da que desejavam seus usuários), o burocrático (garantindo que a definição da estrutura fique em conformidade com as regras em vigor), o consultor (fornecendo aos usuários as opções e soluções sob medida 
para as situações), o assistente administrativo (administrando e simplificando a manutenção de registros e divulgação de dados) e o do demitido (podendo ser temporariamente rejeitado para um uso posterior).

O que se depreende de Askenäs e Westelius (2003), dando uma nuance mais socialmente construída, é a tentativa de contribuir com a discussão de usos de sistemas de informação na mudança organizacional, com uma lógica onde se procura saber qual forma de ação se quer implementar para o sistema de informação ajudar a organização.

Nessa lógica, Laudon e Laudon (20I4) fazem menção que além das tecnologias e das organizações, uma das dimensões dos sistemas de informação são as pessoas que, capacitadas, motivadas e engajadas, contribuirão para que a mudança de pensar e das próprias rotinas organizacionais ocorram, minimamente, como é espectável.

Mas para desempenhar melhor o seu papel, os sistemas de informação são tipificados genericamente em função do nível da organização onde estes se enquadram, funcionam e são utilizados. Assim, de acordo com a forma como são usados e o retorno dado ao processo de tomada de decisão, os sistemas podem ser de contexto operacional ou gerencial, ou seja, resumem-se a sistemas de apoio às operações e sistema de apoio gerencial (LAUDON; LAUDON, 20I4; BAZZOTTI; GARCIA, 2006; BEHL; O’BRIEN; MARAKAS, 20I9).

Em relação aos sistemas de apoio às operações, o seu papel é processar transações eficientemente, controlar processos industriais, apoiar comunicações, colaborações e atualizar bancos de dados da empresa, podendo ser chamados de sistemas de processamento de transações, sistemas de controle de processos e sistemas colaborativos para produtividade de equipes e grupos de trabalho (REZENDE, 2002; LAUDON; LAUDON, 20I4; BEHL; O'BRIEN; MARAKAS, 20I9).

No que se refere aos sistemas de apoio a decisão, de acordo com Behl, O’brien e Marakas (2019), são aqueles que se concentram em fornecer informação e apoio aos gerentes em sua tomada de decisão eficaz, podendo tipificar-se em sistemas de informação gerencial (informações para os gerentes), sistemas de apoio à decisão (para o processo de decisão dos gerentes) e sistemas de informação executiva (informações críticas em quadro de fácil visualização dos executivos).

Mas o avanço tecnológico e as necessidades organizacionais por maior eficácia, eficiência e efetividade, principalmente na componente de informação e conhecimento, permitiram a existência de sistemas de informação com objetivo básico de integrar, consolidar e aglutinar todas as informações necessárias para a gestão organizacional. Esses são chamados de Sistemas Integrados de Gestão Empresarial, mais conhecidos por Enterprise Resource Planing - ERP (ALLEN; KERN, 200I; WAGNER; MONK, 2008; TENHIÄLÄ, RUNGTUSANATHAM; MILLER, 20I8) que no seu estágio final de evolução e integração poderão aglutinar todos os sistemas de informação da entidade e até de outras organizações (SOUZA; SZAFIR-GOLDSTEIN, 2005), transitando para os Langenwalter (I999) chamou de Total Enterprise Integration - TEI. 


\section{A MUDANÇA ORGANIZACIONAL}

O ambiente contemporâneo de vida e negócios das organizações tem sido marcado por uma dinâmica competitiva extremamente agressiva, em que a capacidade de monitoramento permanente do mercado e a sensibilidade quanto a mudanças de hábitos e necessidades dos clientes, bem como à incorporação dessas alterações nos produtos ou serviços da organização podem significar o sucesso ou o insucesso organizacional (RODRIGUES; PINHEIRO, 2005).

Esse realidade descrita solicita um olhar mais roliço das organizações sobre suas rotinas no ambiente interno e sobre demandas do ambiente externo, carecendo então de sempre que possível, se apetrechar de informações atempadas para reagir eficazmente.

Assim, se as rotinas que determinam o funcionamento da organização demonstrarem uma falha, naturalmente ter-se-á que partir para uma mudança ou, simplesmente, visualizar a mudança. Nessa lógica, segundo Weick e Quinn (I999), a mudança ocorre como resposta a uma identificada falha, podendo ser episódica ou contínua e, reconhecendo que as rotinas estão sempre acontecendo, o processo de mudança em si, nunca cessa - vai se adaptando.

Sendo mais generalista, Perazza (20II) afirma que a mudança organizacional é qualquer alteração planejada (ou não) na relação entre a organização e o ambiente, visando sempre à eficiência, eficácia organizacional, garantia da satisfação do cliente e vantagem perante seus concorrentes.

Lippert e Davis (2006) sugerem que, sendo sistemas (ou em referencia aos seus subsistemas), mudança organizacional pode ser a conversão de um sistema em outro, mesmo que nem sempre signifique que seja identificável esse processo ou que parta de uma iniciativa de mudança.

O que sobressai, é que a mudança organizacional é um importante recurso para a organização, sendo um processo em que a mesma otimiza seu performance para um estado desejável, ocorrendo como uma reação ao ambiente, a uma situação de crise, ou a um problema de liderança informado (CHEN, et al., 20I0; BILHIM, 20I0), conforme se representa na figura 5. Mas a esse respeito, Cunha e Rego (20I0) referem que não se pode limitar a percepção de que toda mudança é planejada, pois ocorrem também mudanças emergentes.

Figura 5. Identificação de mudanças ou sua necessidade por via de um sistema integrado de informação

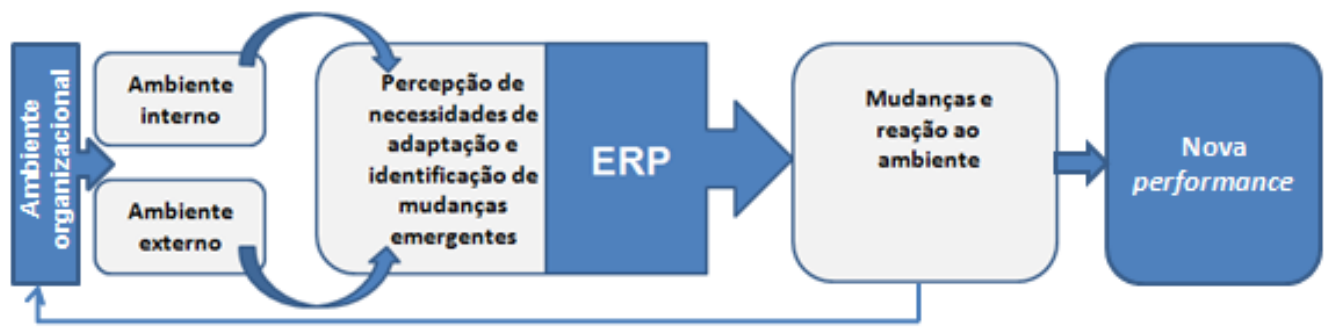

Fonte: Baseado em Rodrigues e Pinheiro (2005), Chen et al., (2010) e Bilhim (20I0). 
Assim, mudança organizacional pode ser entendida como o momento de uma organização em que ela parte do estágio corrente para um futuro e esperançoso estado, que não ocorre espontaneamente, mas em função da sobreposição de forcas para a mudança (internas ou externas) sobre as forcas contra a mudança (PIERCE; GARDNER; DUNHAM, 200I), das quais a organização sempre deve estar a par.

Neste diapasão, a mudança pode ser percebida como um desenvolvimento processual ou um processo que ocorre de forma progressiva (VEN; POOLE, I995) dando resposta, ou sendo resultado de aspectos vindos de fora da organização (forcas para a mudança), que podem ser de natureza política, tecnológica, cultural, demográfica, económica ou de mercado (ALVESSON; SVENINGSSON, 2008).

Acrescentando, Chen, et al.(2010) dissertam que uma mudança bem sucedida não é meramente um processo de ajustamento, mas também requer capacidades de gestão, surgindo deste modo a visão de mudança organizacional proactiva, que ocorre, na maioria das vezes, como consequência de mudança de poder.

E se Chen, et al.(20I0) referem que a mudança precisa ser ajustada a real situação do contexto, vezes há, em que, de acordo com Alvesson e Sveningsson (2008), a mudança ocorre porque simplesmente são desenvolvidos planos de mudança por mera questão de moda.

No que toca a tipificação de mudanças, de acordo com Pierce, Gardner e Dunham (200I), estas podem ser reativas, como resultado de pressões no ambiente que limitam a organização a opção de mudança apenas, ou proactivas, quando a própria organização conclui que a mudança é algo desejável, sendo oposto da mudança por necessidade.

É assim que se reconhece que uma mudança pode se inspirar em situações informadas ou apenas porque os tomadores de decisão concluíram ser, esse novo estágio, o mais adequado para a organização, conforme se resume na figura 6 .

Figura 6. Nuances da ocorrência de mudanças

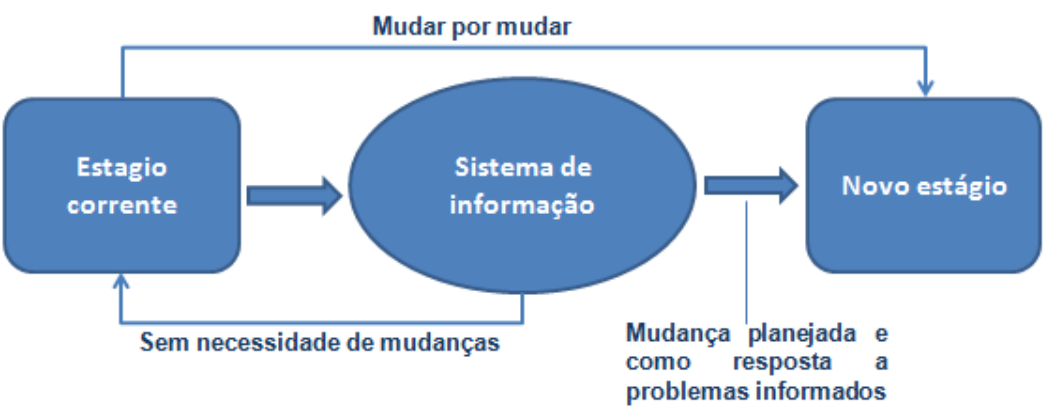

Fonte: Baseado em Alvesson e Sveningsson (2008) e Chen, et al.(2010)

Já para Alvesson e Sveningsson (2008), as mudanças podem apresentar-se como processos evolutivos, significando mudanças operacionais que afetam parte da organização e ocorrem 
dentro da estratégia e da cultura organizacional existentes ou de revolução, sendo de grande escala e afetando radicalmente a cultura organizacional, os sistemas de controle gerencial, a estrutura organizacional, os sistemas de remuneração e a liderança.

Assim, a escala de mudança é por vezes relacionada ao ritmo das mudanças. Mudança revolucionária é geralmente vista como mudança descontínua, enquanto mudança evolutiva é vista como contínua e gradual (WEICK; QUINN, I999; ALVESSON E SVENINGSSON, 2008).

Pierce, Gardner e Dunham (200I), apresentam como fases da mudança a identificação do caminho a seguir; a planificação da implementação (sendo importante nessa fase a informação que deve ser suficiente garantir acerto); a implementação (que inclui competição entre as forcas a favor e as resistências a mudança, bem como a partilha de informação de como se vai processar a mudança) e; a avaliação dos resultados e coleta de feedbacks.

Numa outra vertente, Bilhim (20I0) refere que uma mudança deve passar por processo de descongelamento, de mudança e posterior processo de congelamento, dando espaço para que as rotinas sejam redefinidas e impregnadas pelos atores organizacionais. E fortuitamente, esta acepção se aproxime da trazida por Lippert e Davis (2006) que falam de mudança como conversão de um sistema em outro, neste caso, que se proponha a dar melhores respostas as demandas.

\subsection{Os sistemas de informação e a mudança organizacional}

De acordo com Belle, Nash e Eccles (20I0), o sucesso de um sistema de informação depende de como ele será usado, devendo se perceber com maior antecedência se os empregados ficaram felizes para incorporar em suas atividades de processamento de dados; se o sistema irá fornecer as informações que eles precisam; se estará garantida a integridade da informação.

Fell e Dornelas (2013) propõem que para que se assistam melhores respostas da organização às dinâmicas do ambiente, as organizações devem se apetrechar de sistemas de informação, permitindo uma eficiente disponibilização e utilização de informações e conhecimentos para tomada de decisões estratégicas, táticas ou operacionais. E isso propõe a redefinição de rotinas organizacionais.

Assim, não se pode protelar que as velhas rotinas de tomada de decisão discricionárias e fora de um sistema integrado de informação permaneçam vivas. É então, nessa ótica, que Belle, Nash e Eccles (20I0) afirmam que, embora cuidadosa, a implementação de sistemas de informação pode encarar a potencialidade de surgirem problemas associados ao seu uso no mundo real, propondo que as organizações desenvolvam uma estratégia de gestão de sistemas de informação para que a mudança não seja infrutífera, ou para que não ocorram as inércias (KEEN, I98I) de, sob pressão, os tomadores de decisão preterirem a informação oferecida pelos sistemas de informação. 
Ademais, para amenizar a resistência à mudança e permitir que os sistemas de informação ajudem a organização em processos nesse sentido, sugere-se que, além do treinamento necessário para familiarização com novas formas de actuação perante as novas rotinas, durante a fase de análise de sistemas, os usuários (componente humana da mudança) sejam envolvidos na identificação de problemas que ocorrem em processos e sistemas já existentes, e dada a oportunidade de expressarem suas próprias necessidades de informação (KEEN, I98I; BELLE; NASH; ECCLES, 20Io).

Assim, se os usuários estão cientes de deficiências nos processos do antigo sistema (baseado ou não em computador) e de como estes serão aliviados quando o novo sistema for implementado, então eles estarão mais propensos a aceitar a alteração.

Posto que eventualmente ocorram resistências a mudança, as organizações precisarão de, para usar sistemas de informação, planificar seus sistemas de modo a incorporar adaptabilidade para a mudança, uma vez que de acordo com Avgerou (200I), determinadas organizações podem até ter sistemas de informação, mas os mesmos não reportarem informações estratégicas necessárias para que a organização possa dar respostas coerentes ao ambiente e a competitividade.

Seguindo mesma logica, Campos e Cazarini (2008) rematam que há sempre a necessidade da integração dos sistemas de informação à organização com o propósito de tornar o sistema organizacional mais flexível no momento em que o ambiente competitivo onde as empresas estão inseridas exige mudanças.

Mas fazer essa integração não é automático e nem um mecanismo fácil. É pertinente uma planificação virada para os sistemas de informação ou que esteja neles baseada. E como se percebe de Porto e Bandeira (2006), a definição de um bom panejamento de sistemas de informação é extremamente importante para que possa auxiliar os gestores no processo de tomada de decisão. E em processos de mudança organizacional em que o sistema de informação é a causa ou o instrumento de apoio para responder as contingência, essa planificação deve ser mais acentuada.

Por tanto, o estágio de desenvolvimento atingido pelas tecnologias e pelos aplicativos que podem ser utilizados para compor o sistema de informação de uma organização é capaz de oferecer oportunidades para a existência de uma inter-conectividade entre clientes, fornecedores e organização (LAUDON; LAUDON, 20I4). Este fato tem causado possibilidades de mudança na forma de se organizar e de administrar as organizações. O modelo tradicional baseado em uma estrutura especialista, hierarquizada e centralizada ainda se mantém na cultura organizacional de muitas empresas, mas um novo modelo com estrutura mais enxuta, mais generalista, mais flexível e descentralizada começa a surgir, conforme afiançam os autores anteriormente citados.

Em concordância, num estudo sobre desempenho das tecnologias de informação e mudança organizacional, Rodrigues (2006) garante que a adoção da tecnologia de informação possibilita a redefinição das fronteiras organizacionais e das relações inter-organizacionais com 
clientes e fornecedores, levando à constituição de redes de cooperação e ao desenvolvimento da capacidade de resposta das organizações às mudanças do ambiente. Continuando, o mesmo autor afirma que a utilização da TI provoca a emergência de novos modelos organizacionais desenvolvidos a partir da cooperação interempresarial, alianças estratégicas e redes de empresas, permitindo às organizações reagir com rapidez às variações ocorridas nas exigências dos clientes e no desenvolvimento dos negócios.

Figura 7. Mudanças em organizações com sistemas de informação interconectados.

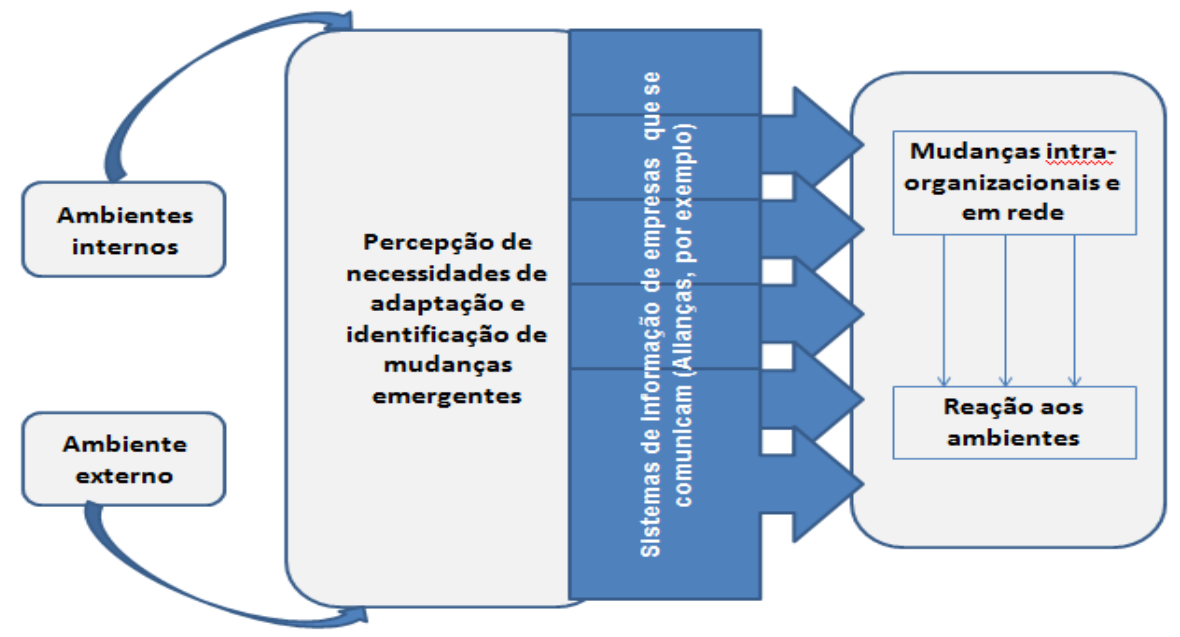

Fonte: Baseado em Rodrigues (2006).

Campos e Cazarini (2008), em seu estudo sobre a integração de sistemas de informação gerencial à organização concluem que a organização faz parte em um sistema vivo que apresenta constantes mudanças, sendo que a tecnologia de informação aliada aos sistemas de informação se torna peça vital para que a empresa possa se manter informada, em função das necessidades de informação em tempo hábil e com confiabilidade como parte integrante do processo de tomada de decisão.

Esta necessidade de informação mencionada é o ponto-chave na justificativa de que o sistema de informação deve estar integrado à organização, formando com ela um sistema único, devendo, na fase de desenvolvimento do sistema da informação e escolha da tecnologia serem considerados o máximo de fatores inerentes à organização e seu negócio (CAMPOS; CAZARINI, 2008).

Assim, legitima-se a ideia de que já não se pode falar de organização sem pensar no seu sistema (ou sistemas) de informação que, necessariamente, devem ser modelados para acompanhar as demandas que chegam à organização ou emanam de seu interior e, ao mesmo tempo, poderem processar de forma atempada tais demandas, garantindo respostas oportunas.

É nesse sentido que, segundo Pádua (200I), para responder às mudanças constantes no ambiente de negócio, as organizações precisam de sistemas ágeis, sendo que, para garantir que os sistemas tenham esta característica, faz-se necessário que os desenvolvedores possuam uma compreensão mais aprofundada sobre a organização, sua estratégia de mercado, seus objetivos, processos e regras de negócios. 
Então, os sistemas de informação tornam-se a peça vital para que organizações possam se manter informadas em tempo hábil e com confiabilidade, como parte integrante do processo de tomada de decisão em contexto de mudanças, significando que, de acordo com Gaines et al. (20II), tendo poder para coletar, avaliar e disseminar a informação o sistema de informação se refaz como recurso estratégico da organização para melhorar sua vantagem competitiva.

Figura 8. Interligação entre sistema de informação, processo de decisão e mudanças

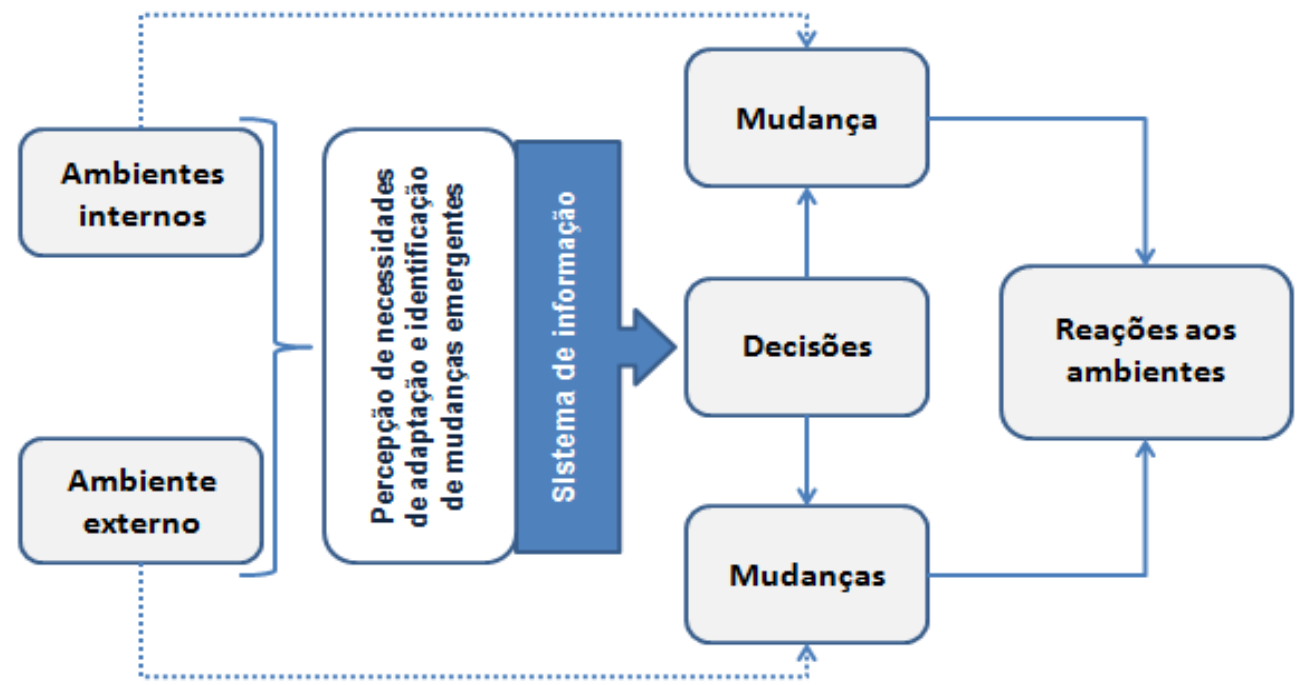

Fonte: Elaborado pelo autor

Conclui-se então que, havendo capacidade de compreender a natureza do ambiente em mudança e as consequências associadas por via de sistemas de informação, melhoram-se processos de tomada de decisão e a reação aos desafios do mercado global, criando assim uma vantagem estratégica, permitindo-lhes mudanças mais próximas do sucesso organizacional (HEMMATFAR; SALEHI; BAYAT, 20I0; GAINES et al, 20II). E a evolução dos sistemas de informação, consoante as necessidades que se espera serem satisfeitas por estes, principalmente no atendimento as constantes mudanças e busca de repostas adequadas e oportunas, faz com que se assistam em organizações, cada vez mais implantados sistemas que se consideram inteligentes (ALMEIDA; HIRATA, 20I6).

Contudo, é sempre importante, no âmbito da mudança organizacional, considerar os entraves que devem ser percebidos na fase do planejamento e, posteriormente, avaliados na implementação das mudanças de modo que, por informação emanada dos sistemas instituídos, sejam mitigados (KEEN, I98I; GAINES et al, 20II).

\section{Considerações finais}

A atua realidade das organizações não permite falar delas sem falar do ambiente que as rodeia, marcado por constantes mudanças. Isto é, elas se afiguram como sistemas abertos e de interligações variadas, de tal modo que a busca de informações para que os gestores tomem melhores decisões inerentes a esse contexto de mudanças deixa de ser uma opção e passa a ser uma necessidade que só pode ser garantida por sistemas de informação eficazes. 
Para um sistema de informação ser eficaz, num contexto de mudança organizacional, ele deve fornecer as informações necessárias. Para que forneça as informações necessárias, este sistema devera ser desenhado ou desenvolvido tendo em atenção as necessidades dos usuários. Assim, uma mudança planificada no seio da organização terá que necessariamente englobar uma planificação estratégica de sistema de informação que alinhe as informações a fornecer com as necessidades de informação para decisão, conforme já afiançavam alguns autores (LEDERER; SETHI, I988; TIDD; BESSANT; PAVITT, 2005).

No entanto, em processo de mudanças, é normal que se visualize resistências ao processo, principalmente num contexto onde as rotinas tendem a garantir um padrão de processos. Mas estas resistências também devem ser percebidas ou coletadas pelos sistemas de informação e remetidas como situações do ambiente interno que merecem dos gestores, decisão cabível. Então, uma planificação mais cuidadosa da mudança poderá envolver todos os membros, recursos e conhecimento da organização, proporcionando sistemas de informação que atendam as expetativas dos usuários, não sendo, neste caso, a sua implementação, um choque para as rotinas organizacionais, mas sim um incremento que irá dinamizar as ações e proporcionar maiores níveis de eficiência e eficácia.

A base de coleta de material para este ensaio foi estritamente documental. Assim, inexistindo suporte empírico para agregar melhores conclusões neste trabalho, entende-se que o mesmo não esgota nas preposições trazidas, mas concomitantemente, finaliza-se com a reafirmação de que, de fato, há um papel excelso a ser desempenhado pelos sistemas de informação para as organizações que, nos dias que correm, estão em constantes mudanças, independendo se são grandes ou pequenas. As abordagens com que se mergulha nesta temática podem ser no âmbito de estudos de tecnologia, inovação, estratégia, estrutura, gestão de informação ou outra, mas fica vincado que uma procura de alinhamento entre os sistemas de informação e a mudança organizacional tende a se afirmar como moda.

\section{Bibliografias}

ABBASI, A.; SARKER, S.; CHIANG, R. H. L. Big data research in information systems: toward an inclusive research agenda. Journal of the association for information systems, Atlanta, I7, n. 2, fev. 20I6. i-xxxii. Disponivel em: <https://search.proquest.com/ docview/I773772634?accountid=I49052>. Acesso em: 30 jul. 2018.

ALLEN, D.; KERN, T. Enterprise resource planning implementation: Stories of power, politics, and resistance. In: RUSSO, N. L.; FITZGERALD, B.; DEGROSS, J. I. Realigning Research and Practice in Information Systems Development: the social and organizational perspective. Boston: [s.n.], 200I. p. I49-I62. Disponivel em: <https://link.springer.com/content/ pdf/Io.I007/978-0-387-35489-7_II.pdf>. Acesso em: 6 ago. 2018.

ALMEIDA, F. C. D.; HIRATA, P. Entendendo e implantando um sistema de inteligência competitiva. REGE - Revista de Gestão, 23, n. 2, 4-6 20I6. III-I22. Disponivel em: <https:// www.sciencedirect.com/science/article/pii/Si8092276I6300IIX>. Acesso em: 2I abr. 2020. 
ALVESSON, M.; SVENINGSSON, S. Changing Organizational Culture: cultural change work in progress. London: Routledge, 2008. 209 p. Disponivel em: <http://untag-smd.ac.id/ files/Perpustakaan_Digital_2/ORGANIZATIONAL\%20CULTURE\%20Changing\%20 Organizational.pdf $>$. Acesso em: 6 ago. 2018.

ANDERSON, D. L. Organization Development: The process of leading organizational change. 4. ed. London: SAGE, 2017.

ASKENÄS, L.; WESTELIUS, A. Five Roles of an Information System: A Social Constructionist Approach to Analysing the Use of ERP Systems. Informing Science, 6, 2003. 209-220. Disponivel em: $<\mathrm{http}: / / \mathrm{www}$. inform.nu/Articles/Vol6/v6p209-220.pdf $>$. Acesso em: 2I out. 2019.

AVGEROU, C. The significance of contextininformation systems. Information SystemsJournal, II, n. I, 200I. 43-63. Disponivel em: <http://eprints.lse.ac.uk/2570/I/SignifiganceofcontextPDF. pdf>. Acesso em: 6 ago. 2018 .

BAZZOTTI, C.; GARCIA, E. A importância do sistema de informação gerencial na gestão empresarial para tomada de decisões. Ciências Sociais Aplicadas em Revista, 6, n. II, 2006. I-I8. Disponivel em: $<$ http://saber.unioeste.br/index.php/csaemrevista/article/view/368>. Acesso em: 27 jan. 2019.

BECKER, M. C. Organizational routines: a review of the literature. Industrial and corporate change, I3, n. 4, ago. 2004. 643-678. Disponivel em: <file://C:/Users/user/Google\%2oDrive/ PROPAD\%2020I8/Analise\%20Organizacional/Ensaio/Fontes/dtho26.pdf>. Acesso em: 25 jul. 2018.

BEHL, R.; O’BRIEN, J. A.; MARAKAS, G. M. Management Information Systems. II. ed. [S.1.]: McGraw-Hill Education, v. Especial Indian Edition, 20I9. 824 p. Disponivel em: $<$ https:// books.google.com.br/books?id=wiSgDwAAQBAJ\&printsec $=$ frontcover\&dq $=\mathrm{O} \% 27 \mathrm{brien},+\mathrm{Jam}$ es + A.\&hl=pt-BR\&sa $=X \& v e d=0 a h U K E w i 8 h a n P{ }_{3} P r o A h X-J 7 k G H a 57 D z A Q 6 A E I M z A B \# v=o n$ epage $\& q \& \mathrm{f}=$ false $>$. Acesso em: $2 \mathrm{I}$ abr. 2020.

BELLE, J.-P. V.; ECCLES, M.; NASH, J. Discovering Information Systems: an exploratory approach. Cape Town: University of Cape Town, 20I0. 175 p. Disponivel em: $<$ https://open.uct.ac.za/bitstream/handle/II427/4I52/Discovering Information Systems. pdf? sequence $=\mathrm{I} \&$ isAllowed $=\mathrm{y}>$. Acesso em: 2I out. 2019.

BEUREN, I.; MACOHON, E. Institucionalização de hábitos e rotinas na contabilidade gerencial à luz da teoria da contigência: um estudo em indústrias de móveis em São Bento do Sul. Revista portuguesa e brasileira de gestão, IO, n. I-2, jan. 20II. 78-9I. Disponivel em: <http://www. scielo.mec.pt/scielo.php?scielo.php?script=sci_arttext\&pid=SI645-446420II000I00008\&lng=pt $\&$ tlng=en>. Acesso em: 27 jul. 2018.

BILHIM, J. A. D. F. Mudança Organizacional. In: COSTA, S. G. D.; VIEIRA, L.; RODRIGUES, J. N. Gestão da mudança. São Paulo: Atlas, 20Io. Cap. I, p. 5-I9.

BONCOMPTE, M. The expected value of perfect information in unrepeatable decision- 
making. Decision Support Systems, Barcelona, mar. 20I8. II-I9. Disponivel em: $<$ https://www. sciencedirect.com/science/article/pii/Sor679236I8300496>. Acesso em: 22 out. 2019.

BORGES, M. E. N. A informação como recurso gerencial das organizações na sociedade do conhecimento. Ciência da Informação, 24, n. 2, Agosto 1995. Disponivel em: <http://revista. ibict.br/index.php/ciinf/article/viewFile/584/586>. Acesso em: I jul. 2016.

BRESSAN, C. L. Mudança Organizacional: uma visão gerencial. SEMINÁRIO DE GESTÃO DE NEGÓCIOS. Curitiba: FAE. 2004. p. I4.

CAMPOS, R. R.; CAZARINI, E. W. Integrando o sistema de informação gerencial à organização: aspetos da modelagem organizacional segundo a metodologia EKD. $4^{\circ}$ CONGRESSO BRASILEIRO DE SISTEMAS. São Paulo: [s.n.]. 2008. p. Io.

CAPEÇA, G.; SÁ-SOARES, F. D. Auditoria do Alinhamento entre Sistemas de Informação e Negócio. XV Conferência da Associação Portuguesa de Sistemas de Informação. Lisboa: [s.n.]. 20I5. p. 66-82.

CARNALL, C. Managing change in organizations. 5. ed. London: Prentice Hall, 2007. 384 p.

CHEN, J.-M. et al. Organizational Change and Development. Organization Science, v. II3, n. I3, p. I-I3, 20IO. Disponivel em: <http://www.nacs.gov.tw/english/_files/I0002I6-30I.pdf>. Acesso em: 6 ago. 2018.

CUNHA, M. P. E.; REGO, A. O tao da mudança: Mudança organizacional desestruturadamente estruturada. In: COSTA, S. G. D.; VIEIRA, L.; RODRIGUES, J. N. Gestão da mudança. São Paulo: Atlas, 20I0. Cap. 2, p. 20-32.

DELLAGNELO, E. L.; MACHADO-DA-SILVA, C. L. Novas formas organizacionais: Onde se encontram as evidências empiricas de ruptura com o modelo burocrático de organização? Organizações \& Sociedade, Salvador, 7, n. 19, dez. 2000. 19-33. Disponivel em: <http://www. scielo.br/scielo.php?script=sci_arttext\&pid=SI984-92302000000300002\&ing=en\&nrm=iso $>$. Acesso em: 30 jul. 2018.

FACCHINI, A. R.; VARGAS, L. M. Sistema de informação em uma organização do setor público. Revista de Administração, São Paulo, 27, n. 3, jul. 1992. 37-47. Disponivel em: $<$ http://200.232.30.99/busca/artigo.asp?num_artigo=447>. Acesso em: 20 out. 2019.

FELL, A. F. D. A.; DORNELAS, J. S. Obstáculos ao Uso da Tecnologia da Informação para a Gestão do Conhecimento: um Estudo de Casos Múltiplos. GESTÃ̃. Org - Revista Eletrônica de Gestão Organizacional, Recife, II, n. 3, 20I3. 463-474. Disponivel em: <http://www.revista. ufpe.br/gestaoorg/index.php/gestao/article/viewFile/495/352>. Acesso em: 6 jul. 2016.

FEY, A. F. A era da informação e uma nova maneira de aprender a aprender. Revista eletrônica Ftec, 20I2. I-6. Disponivel em: <http://revista.ftec.com.br/index.php/revista-eletronica-ftec/ article/download $/ 2 / 2>$. Acesso em: 20 abr. 2014. 
FONSECA, L. M.; DOMINGUES, J. P. How to succeed in the digital age? Monitor the organizational context, identify risks and opportunities, and manage change effectively. Management \& Marketing, I2, n. 3, 20I7. 443-455. Disponivel em: <https://www.degruyter. com/downloadpdf/j/mmcks.20I7.I2.issue-3/mmcks-20I7-0027/mmcks-20I7-0027.pdf $>$. Acesso em: 22 out. 2019 .

GRANT, R. M. The resource-based theory of competitive advantage: implications for strategy formulation. Knowledge and strategy, 1999. 3-23. Disponivel em: <s3.amazonaws.com>. Acesso em: 26 jul. 2018.

HEMMATFAR, M.; SALEHI, M.; BAYAT, M. Competitive advantages and strategic information systems. International Journal of Business and Management, 5, n. 7, 20I0. I58I69. Disponivel em: <http://iranarze.ir/wp-content/uploads/20I8/05/9000-English-IranArze. pdf $>$. Acesso em: 6 ago. 2018.

HENDERSON, J. C.; VENKATRAMAN, H. Strategic alignment: leveraging information technology for transforming organizations. IBM systems journal, 38, n. 2.3, I999. 472-484. Disponivel em: $<\mathrm{http}: / /$ search.proquest.com/docview/2224I0770?accountid $=\mathrm{I} 49052>$. Acesso em: 27 jul. 2018.

HENFRIDSSON, O.; MATHIASSEN, L.; SVAHN, F. Managing technological change in the gigital age: the role of architectural frames. Journal of information technology, 29, n. I, 2014. 27-43. Disponivel em: <http://doi.org/IO.IO57/jit.20I3.30>. Acesso em: 30 jul. 2018.

HOWARD-GRENVILLE, J.; RERUP, C. A process perspective on organizational routines. In: LANGLEY, A.; TSOUKAS, H. Handbook of process organization studies. London: SAGE, 20I6. p. 323-337.

KASEMSAP, K. Mastering Intelligent Decision Support Systems in Enterprise Information Management. In: KHOSROW-POUR, M. Intelligent Systems: Concepts, Methodologies, Tools, and Applications. [S.1.]: IGI Global, 20I8. p. 20I3-2034. Disponivel em: <https://www.igiglobal.com/chapter/mastering-intelligent-decision-support-systems-in-enterprise-informationmanagement/205870>. Acesso em: 2 ago. 2018.

KEEN, P. G. W. Information systems and organizational change. Magazine Communications of the ACM, New York, 24, n. I, jan. 198I. 24-33. Disponivel em: $<$ https://dl.acm.org/ft_gateway. $\mathrm{cfm} ? \mathrm{id}=358543 \& \mathrm{ftid}=44900 \& \mathrm{dwn}=\mathrm{I} \& \mathrm{CFID}=60040496 \& \mathrm{CFTOKEN}=\mathrm{bd} 29877 \mathrm{fd} 92 \mathrm{f} 97 \mathrm{do}-$ AB6C7927-B4DE-6776-E99B6560oDiE85DF>. Acesso em: 6 ago. 2018.

LAUDON, K. C.; LAUDON, J. P. Sistemas de informação gerenciais. Tradução de Célia Taniwaki. II. ed. São Paulo: Pearson Education Brasil, 2014.

LEDERER, A. L.; SETHI, V. The implementation of strategic information systems planning methodologies. MIS Quarterly, I2, n. 3, set. 1988. 445-46I. Disponivel em: < https://www.jstor. org/stable/2492I2>. Acesso em: 22 out. 2019.

LIPPERT, S. K.; DAVIS, M. A conceptual model integrating trust into planned change activities 
to enhance technology adoption behavior. Journal of Information Science, 32, n. 5, out. 2006. 434-448. Disponivel em: <https://journals.sagepub.com/doi/pdf/Io.II77/0I65551506066042?casa_ token=eVWFBlC5jqUAAAAA:hZp66DFL2w7xC_9eAfggDSGrIr7-oraPEJaYqZMBoJuBC2g wIvK6J8RooUSoD1Xt3vRs72RQUMyrDDY>. Acesso em: 22 out. 2019.

LÖBLER, M. L. et al. Sistemas de informação e mudança organizacional: estudo de caso em uma instituição federal de ensino superior. X CONGRESSO NACIONAL DE EXCELÊNCIA EM GESTÃO. Rio de Janeiro: [s.n.]. 20I4. p. I5.

MARKUS, M. L.; ROBEY, D. Information technology and organizational change: causal structure in theory and research. Management science, 34, n. 5, maio 1988. 583-598. Disponivel em: $<$ http://www.jstor.org/stable/2632080>. Acesso em: 27 jul. 2018.

MIETTINEN, R.; VIRKKUNEN, J. Epistemic objects, artefacts and organizational change. Organization, London, I2, n. 3, I maio 2005. 437-456. Disponivel em: <https://doi. org/IO.II77/I35050840505I279>. Acesso em: 26 jul. 2018.

MILAGRES, R. Rotinas - Uma Revisão Teórica. Revista Brasileira de Inovação, Io, n. I, jan. 20II. I6I-I96. Disponivel em: <https://periodicos.sbu.unicamp.br/ojs/index.php/rbi/article/ view/86490I3 $>$. Acesso em: 23 out. 2019.

MÜLLER, L. B.; VIEIRA, M. M. F. Rotinas organizacionais defensivas: um estudo sobre as formas de manifestacao durante um processo de mudança organizacional. XXIII ENAMPAD. Foz do Iguaçu: [s.n.]. I999.

NASCIMENTO, C. A. X. D.; RUAS, R. L.; SILVA, D. D. Rotinas organizacionais no ambiente da teoria evolcionária. Revista Ibero-Americana de Estratégia, I6, n. 3, 7-9 20I7. I44-I59. Disponivel em: $<\mathrm{http}: / / \mathrm{www}$. redalyc.org/html/33I2/33I252606oIo/>. Acesso em: 27 jul. $20 \mathrm{I} 8$.

NETO, G. C.; DORNELAS, J. S. Restritividade em sistemas de apoio à decisao e seu impacto na precisão da decisão tomada. Revista ADM.MADE, Rio de Janeiro, I8, n. 2, maio 20I4. I33I54. Acesso em: 30 jul. 2018.

OLIVEIRA, R. R.; OLIVEIRA, R. R.; LIMA, J. B. D. Reflexão sobre a relação entre a mundança de cultura organizacional e a gestão do conhecimento. Perspectiva em Gestão \& Conhecimento, João Pessoa, 6, n. I, jan. 20I6. 19-35. Disponivel em: $<$ http://periodicos.ufpb.br/ ojs2/index.php/pgc>. Acesso em: 30 jul. 2018.

OLSZAK, C. M.; BARTUś, T.; LOREK, P. A comprehensive framework of information system design to provide organizational creativity support. Information \& Management, 55, n. I, jan. 20I8. 94-I08. Disponivel em: <https://www.sciencedirect.com/science/article/abs/pii/ So378720617303300>. Acesso em: 22 out. 2019.

PÁDUA, S. I. D. D. Investigação do processo de desenvolvimento de software a partir da modelagem organizacional, enfatizando regras do negócio. Escola de Engenharia de São Carlos. São Carlos, p. I56. 200I. 
PEARLSON, K. E.; SAUNDERS, C. S.; GALLETTA, D. F. Managing and Using Information Systems: A Strategic Approach. 6. ed. [S.1.]: Wiley, 20I6. 336 p. Disponivel em: $<$ https://books. google.com.br/books?hl=pt-BR\&lr=\&id=kpAgCwAAQBAJ\&oi=fnd\&pg=PAI\&dq=informa tion+systems+strategic + approach\&ots $=\mathrm{gJ}$ ZZ75SrQ1\&sig=MCoXsVBZDIRHDyQvcfhKIn_$\mathrm{R} 84 \mathrm{H} \mathrm{v}=$ onepage $\& \mathrm{q}=$ information $\% 20$ systems $\% 20$ strategic $\% 20$ approach $\& \mathrm{f}=$ false $>$. Acesso em: I ago. 2018.

PENTLAND, B. T.; FELDMAN, M. S. Organizational routines as a unit of analyses. Industrial and Corporate Change, I4, n. 5, 26 ago. 2005. 793-8I5. Disponivel em: <watermark.silvercahir. com>. Acesso em: 26 jul. 2018 .

PERAZZA, F. O que é mudança organizacional? Mudança Organizacional, 20II. Disponivel em: $\quad<$ http://mudancaorganizacional.blogspot.com/20II/o3/o-que-e-mudanca-organizacional. html>. Acesso em: 6 ago. 2018.

PEREIRA, M. J. L. D. B.; FONSECA, J. G. M. Faces da Decisão: as mudanças de paradigmas e o poder da decisão. São Paulo: Makron Books, 1997.

PIERCE, J. L.; GARDNER, D. G.; DUNHAM, R. B. Managing organizational change and development. In: Management and organizational behavior: an integrated perspective. I. ed. [S.1.]: Thomson, 200I. p. 627-654.

PORTO, M. A. G.; BANDEIRA, A. A. A importância dos sistemas de informações gerenciais para as organizações. XIII SIMPEP. São Paulo: [s.n.]. 2006. p. I2.

RAMALHO, R. A. S. Tecnologia e Sociedade: Responsabilidades e desafios na era da informação digital. São Carlos: UAB-UFSCar, 20I3. 67 p. Disponivel em: <http://hdl.handle. net/I23456789/2664>. Acesso em: 27 jul. 2018.

REZENDE, D. A. Evolução da Tecnologia da Informação nos Últimos 45 anos. Revista FAE Business, 4, 2002. 42-46. Disponivel em: <https://www.joinville.udesc.br/portal/professores/ pfitscher/materiais/Evolu_o_da_TI.pdf $>$. Acesso em: 6 ago. 2018.

REZENDE, D. A. Engenharia de Software e Sistemas de Informação. 3. ed. Rio de Janeiro: Brasport, v. 3, 2006. 3I6 p. Disponivel em: <https://books.google.com.br/ books?hl=pt-PT\&lr $=\& i d=r t B v l \_L-I m c C \& o i=f n d \& p g=P T 23 \& d q=+$ Engenharia + de + Softwar $\mathrm{e}+\mathrm{e}+$ sistemas $+\mathrm{de}+$ informa $\% \mathrm{C}_{3} \% \mathrm{~A} 7 \% \mathrm{C}_{3} \%$ B5es\&ots=9Ahj5Pot-q\&sig=G-AZHQsQihVVPTs3RiLQaXgEijc\#v=onepage\&q=Engenharia\%20de\%20Software\%20e\%20sistemas\%20 de\%20inform>. Acesso em: 22 out. 2019.

REZENDE, D. A.; ABREU, A. F. D. Tecnologia da informação aplicada a sistemas de informação empresariais. 9. ed. São Paulo: Atlas, 20I3. 376 p.

RODRIGUES, E. O desempenho da tecnologia da informação e as mudanças organizacionais e interorganizacionais. Revista Organizações em Contexto, 2, n. 4, 2006. 50-72. Disponivel em: <https://www.metodista.br/revistas/revistas-ims/index.php/OC/article/view/I356>. Acesso em: 6 ago. 2018 . 
RODRIGUES, E.; PINHEIRO, M. A. S. Tecnologia da informação e mudanças organizacionais. Revista de Informática Aplicada, São Caetano do Sul, I, n. 2, jul. 2005. Disponivel em: $<$ http:// www.ria.net.br/index.php/ria/article/viewFile/4/4>. Acesso em: I9 jul. 2016.

SILVA, H. C. C. D.; ARAUJO, M. A. V.; DORNELAS, J. S. Determinantes de não utilização de frameworks de gestão e/ou governança de IT. Revista gestão e tecnologia, I8, n. 2, maio 2018. 274-299. Disponivel em: $<$ http://search.ebscohost.com/login.aspx?direct $=$ true $\& \mathrm{db}=\mathrm{bsx} \& \mathrm{AN}=\mathrm{I} 3$ 0846877\&lang=pt-br\&site $=$ eds-live $>$. Acesso em: 30 jul. 2018.

SISSON, P.; RYAN, J. J. C. H. A knowledge concept map: structured concept analysis from systematic literature review. Journal of entrepreneurship, management and innovation, I3, n. 3, 2017. 29-70.

SOUZA, C. A. D.; SZAFIR-GOLDSTEIN, C. Tecnologia da Informação aplicada à Gestão Empresarial: Um Modelo para a Empresa Digital. VI SEMEAD /FEA-USP. São Paulo: [s.n.]. 2005 .

SPULDARO, J. D. A institucionalização de rotinas organizacionais. Encontro de estudos em estratégia. Bento Gonçalves: ANPAD. 20I3. p. I-IO.

STAIR, R.; REYNOLDS, G. Fundamentals of Information Systems. 9. ed. Boston: Cengage Learning, 20I7. 560 p. Disponivel em: <https://books.google.com.br/books?hl=pt-BR\&lr=\&id $=$ GtVBDgAAQBAJ\&oi $=$ fnd $\& p g=$ PPI $\& d q=$ the + role + of + information + systems + in + organizatio

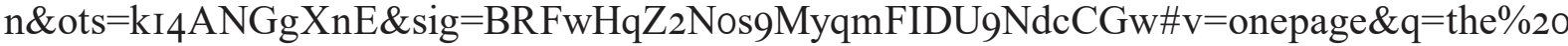
role $\% 200 \mathrm{f} \% 20$ information $\% 20$ systems $\% 20 \mathrm{in} \% 20$ organization $\& \mathrm{f}=$ false $>$. Acesso em: I ago. 2018 .

STANCZYK-HUGIET, E. I. Routines in the process of organizational evolution. Management, I8, n. 2, dez. 2014.

SUDDABY, R.; FOSTER, W. M. History and Organizational Change. Journal of Management, 43, n. I, jan. 20I7. 19-38. Disponivel em: <https://journals.sagepub.com/doi/ full/IO.II77/or492063I667503I >. Acesso em: 23 out. 2019.

TENHIÄLÄ, A.; RUNGTUSANATHAM, M.; MILLER, J. W. ERP System versus Stand-Alone Enterprise Applications in the Mitigation of Operational Glitches. Decision Sciences Journal, 49, n. 3, 20I8. 407-444. Disponivel em: <https://www.researchgate.net/profile/Antti_Tenhiaelae/ publication/3I7I84790_ERP_System_versus_Stand-Alone_Enterprise_Applications_in_the Mitigation_of_Operational_Glitches/links/59ddddI5a6fdcc276fbc9caa/ERP-System-versusStand-Alone-Enterprise-Application>. Acesso em: 6 ago. 2018.

TIDD, J.; BESSANT, J.; PAVITT, K. MANAGING INNOVATION. 3. ed. Chichester: John Wiley \& Sons Ltd, 2005. 602 p. Disponivel em: <https://s3.amazonaws.com/academia. edu.documents/37285072/0470093269.pdf?response-content-disposition=inline $\% 3 \mathrm{~B} \% 20$ filename\%3DManaging_Innovation_Book_for_reference.pdf\&X-Amz-Algorithm=AWS4HMAC-SHA256\&X-Amz-Credential=AKIAIWOWYYGZ2Y53UL3A\%2F20I9I022\%2Fus-e>. Acesso em: 22 out. 2019. 
VASCONCELOS, A. et al. Arquitectura de Sistemas de Informação: A Ferramenta de Alinhamento Negócio / Sistemas de Informação? III CONFERÊNCIA DA ASSOCIAÇÃO PORTUGUESA DE SISTEMAS DE INFORMAÇÃO. Porto: [s.n.]. 2002. p. I8.

VEN, A. H. V. D.; POOLE, M. S. Explaining Development and Change in Organizations. Academy of Management Review, 20, n. 3, jul. 1995. 5IO-540. Disponivel em: $<$ https://journals. aom.org/doi/abs/I0.5465/amr.1995.9508080329>. Acesso em: 6 ago. 2018.

VIET, N. Q.; BEHDANI, B.; BLOEMHOF, J. The value of information in supply chain decisions: A review of the literature and research agenda. Computers \& Industrial Engineering, I20, jun. 20I8. 68-82. Disponivel em: <https://www.sciencedirect.com/science/article/pii/ So3608352I830I76I>. Acesso em: I ago. 2018.

VOLKOW, N. Strategic use of information technology requires knowing how to use information. In: AVGEROU, C.; WALSHAM, G. Information technology in context. New York: Routledge, 20I7. p. $72-85$.

WAGNER, B.; MONK, E. Concepts in Enterprise Resource Planning. 3. ed. Boston: Cengage Learning, 2008. 272 p.

WEICK, K. E.; QUINN, R. E. Organizational change and development. Annual Review of Psychology, 50, 1999. 36I-386. Disponivel em: <https://www.annualreviews.org/doi/abs/Io.II46/ annurev.psych.50.I.36I>. Acesso em: 6 ago. 2018. 